\title{
Ibuprofen encapsulation by eudragit RSI00 as microspheres: preparation and drug release
}

\begin{abstract}
Eudragit RS100 microspheres containing Ibuprofen as an example for acidic drugs was prepared using solvent evaporation technique. It was found that the using of $3 \%$ gelatin as an anti aggregating agent in the $0.1 \mathrm{~N} \mathrm{HCl}$ aqueous phase is essential to prevent the appearance of the drug crystals in the aqueous phase during preparation. Also the formulation was individual small particle size microspheres with low mean particle size $(37 \mu \mathrm{m})$. The prepared microsphere showed the presence of drug crystal attached to the microspheres surface which supports the anti aggregating mechanism of gelatin. Gelatin is hydrophilic colloids forming multi-molecular films around the emulsified droplets resulting resist the coalescence of the emulsified microspheres and hindering the rapid diffusion of the organic phase to the aqueous phase which lead to appearance of the drug crystals swimming in the external phase during preparation. The maximum amount of drug release in $0.1 \mathrm{~N} \mathrm{HCl}$ was $4 \%$ in two hrs. The drug release process in phosphate buffer $\mathrm{pH} 6.8$ showed rapid initial and incomplete drug release $(45 \%$ of actual drug content in $9 \mathrm{hr})$. This is nearly normal of all products prepared by solvent evaporation technique. DSC showed the drug is encapsulated in three forms, solid solution form, drug crystal form and other kind of physico chemical interaction with the polymer. The burst effect may be due to the drug crystals attached at the surface of the microspheres in addition to molecular dispersion of the drug near the microsphere surface. Incomplete drug release may be due to physico chemical drug interaction with the polymer and/or perfectly drug crystal encapsulated by the polymer.
\end{abstract}

Keywords: eudragit RS100, ibuprofen, solvent evaporation technique, burst effect, incomplete drug release
Volume 4 Issue I - 2017

Omar Mady
Pharmaceutical Technology Department, Tanta University, Egypt

Correspondence: Omar Mady, Pharmaceutical Technology Department, Faculty of Pharmacy, Tanta University, Egypt, Tel |14-18I-966-I,Email omer.mady@gmx.at

Received: June 08, 2017 | Published: October 09, 2017

\section{Introduction}

The success in the preparation of Eudragit RS100 microspheres in 0.IN HCI solution using gelatin as antiaggregating agent, encouraged the selection of an acidic drug to be encapsulated using a solvent evaporation technique. ${ }^{1}$ Ibuprofen is a non-steroidal, antiinflammatory, analgesic and antipyretic drug approved for use as adjunct therapy in the treatment of rheumatoid and osteoarthritis. ${ }^{2}$ Chemically, it is a propionic acid derivative. ${ }^{3}$ It is slightly soluble in water and has a poor wettability. However, it is completely bioavailable and is rapidly absorbed after oral administration. It gives maximum plasma concentration within 2 hours. ${ }^{4}$ The major side effect of Ibuprofen therapy involves gastrointestinal toxicity. Kawashima et al..$^{5}$ prepared controlled release Ibuprofen microspheres using acrylic polymers. An ethanolic solution of Ibuprofen and an acrylic resin was poured into an aqueous medium with stirring. The finely dispersed ethanolic droplet-like coacervates formed in the aqueous phase were gradually solidified and transformed into microspheres during agitation. Kawashima stated that the concentration of drug and polymer and the agitation speed of the system controlled the size of the microspheres. Also, Ibuprofen prolonged-release spherical micrometrics were prepared by using Eudragit $\mathrm{RS}_{\mathrm{I} 00}$ and a novel emulsion-solvent diffusion method. ${ }^{6}$ Examining cross sections of the formed spherical matrix before and after dissolution studying using a scanning electron microscope and a porosimeter indicated that the resultant micro matrix had a sponge-like internal structure. The spherical matrix was successfully recovered with a relatively high concentration of drug in ethanol $(0.4-0.6 \mathrm{gm} / \mathrm{ml})$ and over a wide temperature range $\left(5-35^{\circ} \mathrm{C}\right)$. The size of the spherical matrix could be easily controlled by varying the agitation speed of the system and by adjusting the concentration of the emulsifier added to the aqueous medium. Also in relation to the solvent diffusion procedure it was reported that Eudragit RS100 and RL100 microspheres containing Ibuprofen were prepared and evaluated. The microspheres were prepared by modified quasi-emulsion solvent diffusion method. The author reported when there was an initial difference of temperature between the aqueous phase and dispersed emulsion phases, the yield of preparation was increased distinctly. The drug loading capacities were very high for all formulations of the microspheres obtained. Mean particle size changed by changing the drug-polymer ratio, volumes of solvent or polyvinyl alcohol concentration. The flow properties were much improved over those of the original crystals. ${ }^{7}$

Ibuprofen was also microencapsulated with Eudragit RS using an $\mathrm{o} / \mathrm{w}$ emulsion solvent evaporation technique. Microspheres with good range of particle size can be prepared, depending on the formulation components. The drug: polymer ratio had a considerable effect on the entrapment efficiency. However, particle size distribution of microspheres was more dependent on the volume of chloroform and polyvinyl alcohol concentration rather than the drug: polymer ratio. ${ }^{8}$ Also in relation to the same encapsulation procedure, it was also reported that the emulsion solvent evaporation method was used to prepare Eudragit RSPO microspheres containing Ibuprofen. Microspheres morphology and chemical integrity were studied by a scanning electron microscope and Fourier transforms infrared spectroscopy. The SEM reports depict that the surface morphology changes drastically as the types of polymer combination and drug/ polymer ratio changes. The FTIR shows a successful formulation 
technique of drug and polymer by showing the presence of drug in the microspheres. ${ }^{9}$ It was also tried to evaluate the influence of plasticizer ratio on the drug release rate and physicochemical characteristics of ethyl cellulose microspheres containing Diltiazem and prepared by solvent evaporation technique. Results indicated that the physicochemical properties of microspheres were strongly affected by the drug/polymer ratio and the concentration of plasticizer used in the production technique. The surface morphology and pore volume of microspheres significantly varied based on the plasticizer content in the formulation. ${ }^{10}$ The aim of this work is to prepared Eudragit RS100 microspheres containing Ibuprofen as a model for acidic drug to be encapsulated by the method reported by the author ${ }^{1}$ as a tool to increase the drug content. Also studying the drug release process in relation to the drug entrapment methods.

\section{Materials and methods}

\section{Materials}

Chloroform, Dichloromethane (both were analytical grade), Eudragit RS100 granules (Rohm Pharm, Germany), Gelatin Alba powder, OAB (Pharm Productions, Austria). Ibuprofen (Cairo Pharmaceutical Company, Egypt).

\section{Equipment}

Differential scanning calorimeter (Perkin Elmer DSC 4, USA), Dissolution tester (Erweka type DT, Germany), Mechanical stirrer (Heidolph, Germany), Optical microscope (Ieitz 10000, Wetzlar, Germany), Vibrating set sieve (VEB, Metalweberei, neuatatt Drla, Germany). UV spectrophotometer (Perkin Elmer, Lambda 1, USA).

\section{Preparation of Eudragit RSI00 microspheres containing ibuprofen}

$200 \mathrm{ml}$ of $0 . \mathrm{IN} \mathrm{HCl}$ solution containing different concentrations gelatin as required was stirred at room temperature and at a speed of $320 \mathrm{rpm}$. $100 \mathrm{ml}$ of dichloromethane containing $10 \mathrm{gm}$ of $20 \%$ Ibuprofen and $80 \%$ Eudragit RS100 were added drop wise to the acid solution while stirring. Stirring was continued for 6 hours at room temperature. The resulted microspheres were collected by filtration, washed several times with $0.1 \mathrm{~N} \mathrm{HCl}$ solution and dried at ambient temperature.

\section{Characterization of the prepared microspheres}

Optical microscopy scanning: Morphology of the microspheres were studied by using an optical microscope. Photographs were taken for the microspheres at 40 and 100 magnifications.

Sieve analysis: According to U.S. Pharmacopeia ${ }^{11}$ for testing powder fineness, a definite weight of sample $(20 \mathrm{gm})$ is placed on the proper sieve of a mechanical shaker. The Microspheres were shaken for a definite period of time and the microspheres passed from one sieve and retained on the next finer sieve was collected and weighed.

Determination of drug content of the microspheres: $100 \mathrm{mg}$ of the prepared Eudragit RS100 microspheres containing Ibuprofen was exactly weighed and dissolved in chloroform. The drug content of the solution was determined spectrophotometry at $265 \mathrm{~nm}$ using pure chloroform as a blank since experimentally Eudragit RS100 did not interfere with the drug absorption and the maximum drug absorption $\left(\lambda_{\max }\right.$ in chloroform) determination.
Differential scanning calorimetery: Differential scanning analysis of Eudragit RS100 microspheres, Ibuprofen powder and Eudragit RS100 microspheres containing Ibuprofen were carried out. The heating cycle ranged from 20 to $240^{\circ} \mathrm{C}$.

\section{Drug release from the prepared microsphere}

An exact weight of Eudragit RS100 microspheres containing $100 \mathrm{mg}$ Ibuprofen of different size was added to USP Type 1. The release medium were $500 \mathrm{ml}$ of $0.1 \mathrm{~N} \mathrm{HCl}$ (simulated gastric juice) and $900 \mathrm{ml}$ of phosphate buffer $\mathrm{pH} 7.2$ (simulate intestinal fluid). The temperature was maintained at $37 \pm 0.2^{\circ} \mathrm{C}$ and the stirring rate was adjusted at $200 \mathrm{rpm}$. Samples $(5 \mathrm{ml})$ were withdrawn at predetermined time intervals for $2 \mathrm{hrs}$ of the acid medium and $8 \mathrm{hrs}$ for phosphate buffer $\mathrm{pH}$ 7.2. A fresh release medium was added to replenish each sample withdrawn. The drug content of the sample was determined spectrophotometrically at $222 \mathrm{~nm}$. Three replicates of the entire runs were conducted.

\section{Results and discussion}

The use of a solvent evaporation technique to prepare microspheres containing Ibuprofen was established by adding the organic phase containing drug and polymer to the aqueous phase containing a suitable antiaggregating agent. The mixture was stirred and the formed microspheres were collected by filtration. The success for the preparation of placebo Eudragit RS100 microspheres in acid medium using dichloromethane as the organic phase and $0.1 \mathrm{~N} \mathrm{HCl}$ containing gelatin as the aqueous phase as a tool to increase the drug encapsulation in the microsphere structure encouraged to select Ibuprofen an example for acidic drugs to be encapsulated by this method. ${ }^{1}$ Different gelatin concentrations as anti aggregating agent dissolved in $0.1 \mathrm{~N} \mathrm{HCl}$ as an aqueous phase for the preparation of the microspheres and the mixture was stirred at constant stirring rate (320rpm). Theoretical drug content used was $20 \%$ of $10 \mathrm{gm}$ drugpolymer weight. During preparation of the microspheres using 1 or $2 \%$ of gelatin; drug crystals in the aqueous phase were observed. The concentration of drug crystals in the aqueous phase on using $1 \%$ gelatin was more than that observed on using $2 \%$ gelatin. However, when the gelatin concentration was increased to $3 \%$, the drug crystals disappeared from the aqueous phase during preparation.

In the emulsion solvent evaporation process, a polymer-drug mixture (Eudragit RS100- Ibuprofen 4:1, 10gm) dissolved in the organic phase was emulsified in $0.1 \mathrm{~N} \mathrm{HCl}$. The polymer and the drug deposited as a result of partitioning of the organic solvent between the dispersed phase and the continuous phase; partitioning was continued by removal of the organic solvent through evaporation. ${ }^{12}$ Bodimeir et al. ${ }^{13}$ proved that diffusion and drug loss across the droplet interface occurred only during the first 10 minutes after emulsification of the organic phase in the conventional solvent evaporation. Mariam et al. ${ }^{14}$ on studying the effect of gelatin grade and concentration on the migration of solute into and through glycerogelatin gels stated that micro viscosity is a factor governing diffusion. The gelatin concentration in the aqueous media influenced micro viscosity and hence diffusion. This was believed to be due to dissolution of the water-soluble fractions of gelatin. Takamura et al. ${ }^{15}$ suggested that diffusion of electrolytes to the interior of the microcapsules was a rate determining factor during the permeation process because the formation of a stable diffusion layer of the electrolyte was considered to be highly probable inside the microcapsule. Furthermore, Takamura ${ }^{15}$ studied the effect of membrane materials and the viscosity of the 
aqueous phase on permeability of polyamide microcapsules toward the electrolytes. The author reported that increasing the viscosity of the aqueous phase clearly resulted in a decrease in the rate of sodium chloride entry. It is quite unlikely that polyvinylpyrrolidone molecules, used to increase viscosity of the aqueous medium are adsorbed on the membrane by competing with dispersing agent molecules and offer resistance to the entry of sodium chloride since the former molecules are far less surface active than the latter ones. ${ }^{16}$

Accordingly, it can be concluded that during the emulsification of dichloromethane containing polymer and drug, some dichloromethane containing drug is rapidly diffused to the aqueous medium. Since the drug is insoluble in the aqueous phase, it appeared as drug crystals. The volume of dichloromethane, rapidly diffused to the aqueous phase, depended on the concentration of gelatin used. This could explain the observation of the presence of high amount of drug crystals in the aqueous medium on using $1 \%$ of gelatin compared to on using of $2 \%$ gelatin. The absence of drug crystals in the aqueous phase on using $3 \%$ gelatin may be due to the hindering effect of the concentrated gelatin solution $(3 \%)$ to the rapid diffusion of dichloromethane. Ibuprofen crystals observed on using 1 and $2 \%$ of gelatin in the aqueous phase disappeared completely during washing of the microspheres with $0.1 \mathrm{~N} \mathrm{HCl}$ which is in agreement with what stated by Benita et al. ${ }^{5}$ These findings were also reflected on the drug encapsulated \% which is $97.22 \% \pm 0.667$ in case of using $3 \%$ gelatin, $95.48 \% \pm 0.344$ in case of using $2 \%$ gelatin and $90.5 \% \pm 0.732$ in case of using $1 \%$ gelatin. These results support what stated before about the role of gelatin concentration on appearance and disappearance of drug crystal in the aqueous phase. At the same time morphological study of the prepared microspheres using 40 times magnification showed spherical individual and nearly homogenous product (Figure 1). But surface scanning of the prepared microspheres using higher magnification (100 times) shows drug crystals attached to the surface of the dried microsphere prepared on using 3\% gelatin as an anti aggregating agent (Figure 1).
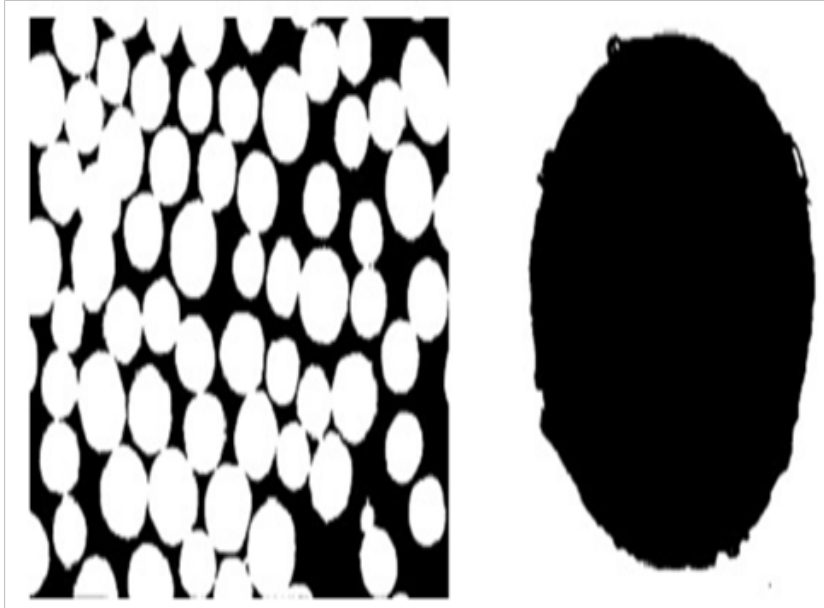

Figure I Morphological and surface photo of the eudragit RSI 00 microspheres containing ibuprofen

These results again support the explanation of the role of gelatin on hindering the rapid diffusion of dichloromethane to the aqueous phase and disappearance of drug crystals in the aqueous phase during microspheres preparation. In addition it may also indicate the inability of the polymer to encapsulate the drug over certain concentration content. The particle size distribution shows a unimodal bell shape structure specially in case of using 3\% gelatin as an anti aggregating agent (Figure 2B). In addition the back tail of the particle size distribution curve decrease in case of using $2 \%$ gelatin than on using $1 \%$ and disappeared on using $3 \%$ support the normally reported about the effect of the antiaggregating agent on decreasing particle size. Also, the mean particle size was found to be decreased with increasing gelatin concentration used which shows a straight line in correlation with gelatin concentrations with $r_{2}$ value of 0.997 (Figure 2A).
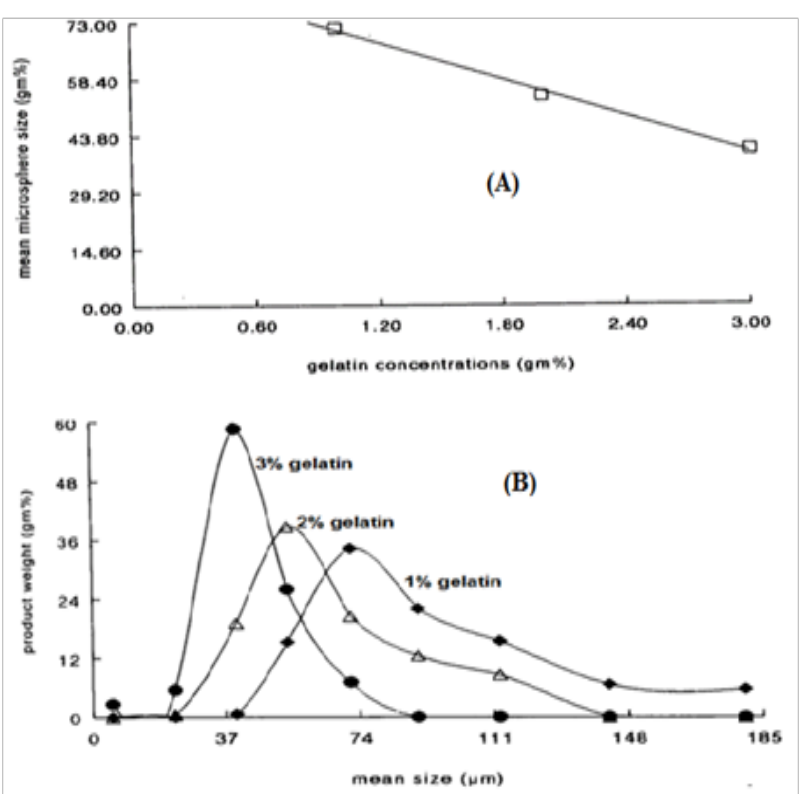

Figure 2 Effect of gelatin concentrations on the microsphere size distribution.

Figure 3A, shows the release rate of Ibuprofen in 0.IN HCI from Eudragit RS100 microspheres. From the figure it can be noticed that the maximum amount of drug released in $0.1 \mathrm{~N} \mathrm{HCI}$ is $4 \mathrm{mg} \%$ which is higher than that the determined Ibuprofen solubility in O.IN $\mathrm{HCl}\left(1.835 \% \pm 0.085\right.$ determined). Kislaiogu et al. ${ }^{17}$ reported that, on carrying out dissolution of the coprecipitated product of Ibuprofen with anionic and (anionic-cationic) Eudragit found a slight but a significant increase in the dissolution of the drug in the acidic media. Although the exact mechanism involved in such behaviour was unknown, the quantitative wetting tests demonstrated instantaneous wetting of the coprecipitated prepared with such polymers. The theoretical explanation could be also supported with what is reported about the poor wettability of the drug. ${ }^{4}$ Also after $45 \mathrm{~min}$, the amount of released drug started to decrease which is clearly noticed from Figure 3B. That is may be due to electrostatic adsorption of the drug on the Eudragit RS100 microspheres.

Figure 4 shows the drug release in phosphate buffer $\mathrm{pH}$ 7.2. From the figure it can be noticed that, nearly $30 \%$ of the drug released as rapid initial drug released. Burst effect is a characteristic phenomenon of the drug released from nearly all microspheres prepared by solvent evaporation technique. ${ }^{18-30}$ There are different explanation for the rapid initial drug release from microspheres prepared by solvent evaporation technique. One of them is, the early fast release as a result of the presence either imperfect microcapsules or due to surfaceadsorbed drug. ${ }^{24,31}$ Jeyanthi et al. ${ }^{30}$ had an image to the microsphere structure and related the initial burst effect to dispersing the drug mainly on the periphery of the microsphere matrix. The interpretation of Jeyanthi et al. ${ }^{30}$ was in agreement with that of Suzuki et al. ${ }^{19}$ since 
the latter related the burst effect to a greet concentration of the drug near the surface of the microspheres. Bodmeier et al..$^{31}$ had an deeper image and related the burst effect to the spongy structure of the microspheres in which the drug was being located within the pores so that drug released from the microspheres occurred mainly by leaching through the pores. Not only that but also, Wada et al. ${ }^{18}$ suggested nearly all related factors affecting the burst effect to the heterogeneous drug dispersion in the polymer matrix. The author scanned of the prepared Eudragit RS100 microspheres containing Ibuprofen and the result shows the presence of drug crystal attached to the surface of the microspheres. Accordingly, it can be concluded that the presence of free drug crystal attached to the microsphere surface in addition to all other reported theory may be responsible about the presence of what is called burst effect.

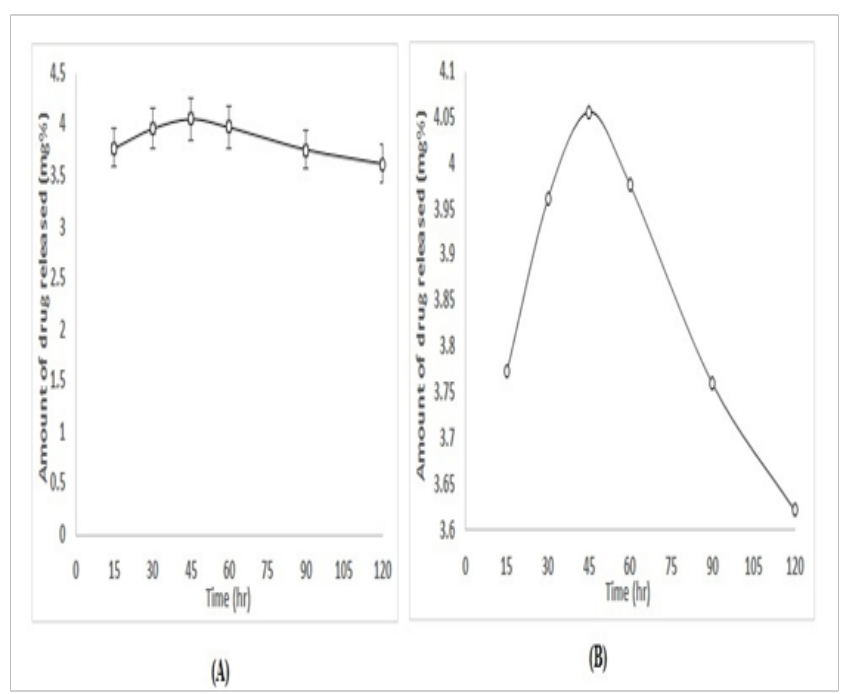

Figure 3 lbuprofen release from eudragit RSI00 microspheres in $0.1 \mathrm{~N} \mathrm{HCl}$.

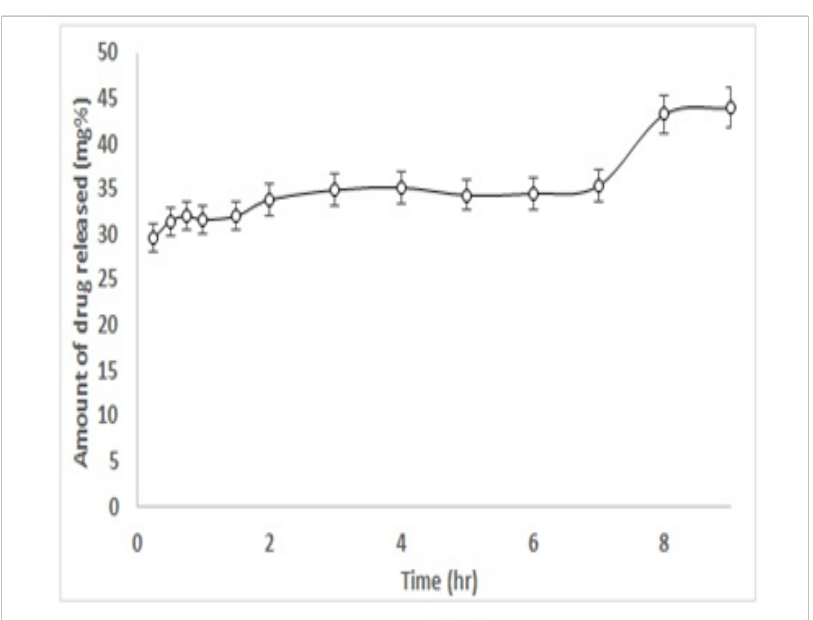

Figure 4 lbuprofen release from eudragit RSI00 microspheres in phosphate buffer $\mathrm{pH} 7.2$.

Release studies revealed that, the highest cumulative drug release after $9 \mathrm{hrs}$ is $45 \%$ indicating incomplete drug release. The incomplete drug release from products prepared by solvent evaporation technique is frequently reported with different trials for explanation. ${ }^{26,32-37}$ For example, the incomplete drug release was considered to be due to be the drug-polymer interaction ${ }^{34,36}$ or due to the retardation capacity of the coating polymer to diffusion of drug. ${ }^{31,32}$ Spenlehaur et al. ${ }^{30}$ related the incomplete drug release to the high solubility of the drug in the polymer matrix or to the sink solution penetration into the microsphere. Accordingly the physico-chemical structure of the microspheres was studied by I.R. and differential scanning calorimetry (DSC) methods.

The scan of pure Ibuprofen showed that in the region of $4000-2000 \mathrm{~cm}^{-1}$ Ibuprofen exhibited a broad band at about 2600 $2800 \mathrm{~cm}^{-1}$ due to stretching of the carboxylic $\mathrm{OH}$ group as a result of intermolecular hydrogen bonding. The aromatic $\mathrm{C}-\mathrm{H}$ stretching appeared as a broad multiple band in the region of $3000-3200 \mathrm{~cm}^{-1}$. A broad multiple band, between $2800-3000 \mathrm{~cm}^{-1}$, is attributed to the aliphatic $\mathrm{C}-\mathrm{H}$ stretch. In the carbonyl frequency region, the drug shows a strong band at $1723 \mathrm{~cm}^{-1}$ due to the $\mathrm{C}-\mathrm{O}$ stretch in the carboxylic group. The $\mathrm{CHx}-$ vibrations at 1463,1381 and $1366 \mathrm{~cm}^{-1}$ can be also seen. The band at $1508 \mathrm{~cm}^{-1}$ indicates the presence of an aromatic group. The strong absorption band at $780 \mathrm{~cm}^{-1}$ is characteristic of the substituted aromatic ring. Finally, the bands at 1420 and $1231 \mathrm{~cm}^{-1}$ are due to coupling between in-plane $\mathrm{OH}$ bending and $\mathrm{C}-\mathrm{O}$ stretching of the dimmer (Figure 5). ${ }^{38}$

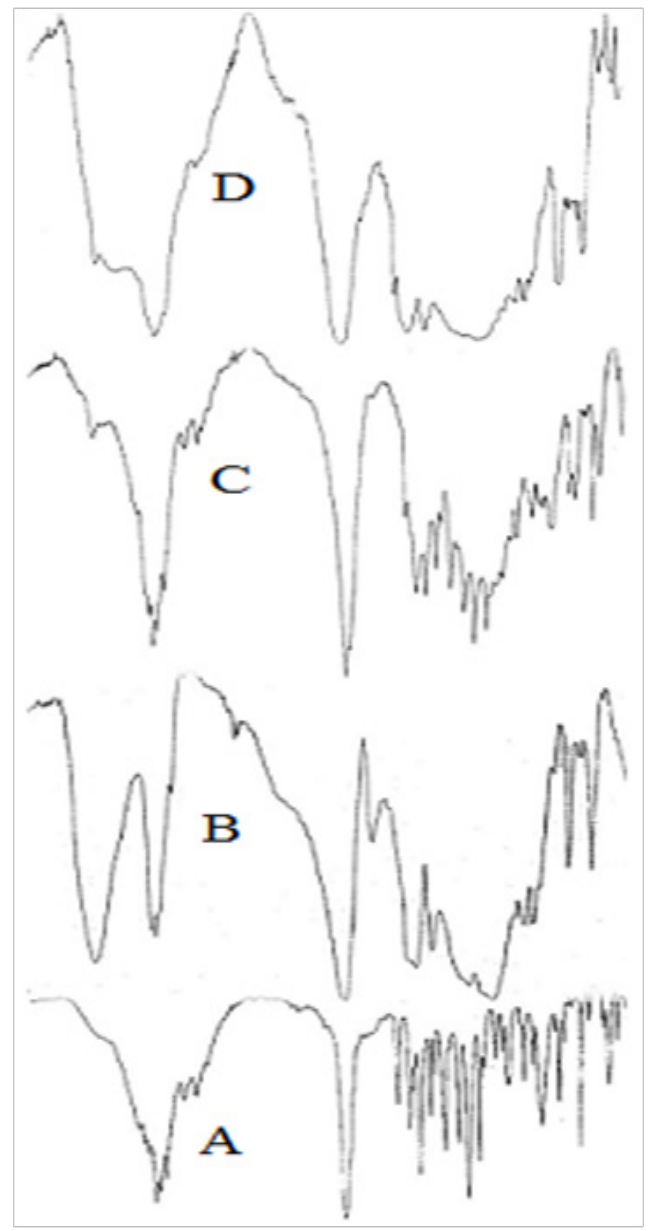

Figure 5 Infra-red scan of
a. ibuprofen;
b. eudragit RSI00;
c. ibuprofen-eudragit RSI00 physical mixture and
d. Eudragit RSI00 microspheres containing Ibuprofen. 
IR spectra of the polymer (Eudragit $\mathrm{RS}_{100}$ ) showed that in the region of 4000-2000 $\mathrm{cm}^{-1}$, the polymer shows a broad band centered at nearly $3440 \mathrm{~cm}^{-1}$ due to the $\mathrm{O}-\mathrm{H}$ stretch from water. The characteristic bands of the ester groups at $1115-1190$ and $1240-1270 \mathrm{~cm}^{-1}$ as well as of the $\mathrm{C}=\mathrm{O}$ ester vibration at $1726 \mathrm{~cm}^{-1}$ were also shown. The $\mathrm{CHx}$ vibrations at $1385,1450,1475$ and $2950-3000 \mathrm{~cm}^{-1}$ can be also seen.

IR scan of physical mixture of Eudragit RS100 and drug showed the additive of the characteristic bands of the drug and the polymer. The I.R. spectra of Eudragit RS100 micro spheres containing Ibuprofen shows that in the region $4000-2000 \mathrm{~cm}^{-1}$. There was a broad band representing the position of moisture $\mathrm{OH}$ group, the aromatic $\mathrm{C}-\mathrm{H}$ stretch, the aliphatic $\mathrm{C}-\mathrm{H}$ stretch and also a broad band for $\mathrm{OH}$ stretching of carboxylic group. Also, a broad band, between 1900 $1600 \mathrm{~cm}^{-1}$, which is sharp peak at $1723 \mathrm{~cm}^{-1}$ representing the carbonyl groups. The presence of drug can be identified from the two small peaks at 1511 and $780 \mathrm{~cm}^{-1}$ which represented the aromatic ring of the drug.

Kislalioglu et al. ${ }^{17}$ found that the spectrum of Eudragit $\mathrm{RS}_{100}-\mathrm{RL}_{100}$ mix after co-precipitation with Ibuprofen indicated that the peak at $1705 \mathrm{~cm}^{-1}$ was considerably reduced and that at $1730 \mathrm{~cm}^{-1}$ was absent which may be due to breakdown of the intermolecular hydrogen bonding of Ibuprofen and establishment of weaker forces in the complex system. The quantitative crystallinity of the co-precipitate using powder $\mathrm{x}$-ray diffraction showed an interaction between the polymer mixture and Ibuprofen. Also EI-Hinnawi et al. ${ }^{39}$ studied the I.R. spectra of Ibuprofen dispersed in PVP and found a change in the spectra of the drug in its dispersed form while the physical mixture showed identical spectra of both polymer and pure drug. Accordingly, it can be suggested that the encapsulation of Ibuprofen by Eudragit RS100 led to change in the total symmetry of the drug molecules the polymer matrix. ${ }^{39}$ Also, the disappearance of the drug peaks in IR microsphere scan may be due to the widespread of the polymer bands which can be clearly observed from IR scan of the physical mixture.

In order to shed more lights on the structure of the micro spheres, DSC studies were carried out for each of Ibuprofen, placebo Eudragit RS100 microspheres, Eudragit RS100 microspheres containing drug (Figure 6). From the figure it can be noticed that, the melting point of pure drug is $79^{\circ} \mathrm{C}$. The Tg of the placebo Eudragit RS100 microsphere prepared in $0.1 \mathrm{~N} \mathrm{HCI}$ using $3 \%$ gelatin as anti aggregating agent is a broad event fall between $45-90^{\circ} \mathrm{C}$ with a maximum at $62^{\circ} \mathrm{C}$. Studies concerning ibuprofen microspheres showed that, the Ibuprofen melting endotherm is diffused and splitted into two parts; one of which having a sharp peak located at the position for pure Ibuprofen, the second one was a broad event fall between $30^{\circ} \mathrm{C}$ and $110^{\circ} \mathrm{C}$. The sharp peak in the figure was considered to be due to free Ibuprofen crystals. Comparing the Tg of Eudragit RS100 in the DSC scan of microspheres containing drug and placebo revealed the complete disappearance of the $\mathrm{Tg}$ of the polymer.

Kislalioglu et al. ${ }^{17}$ studied the crystallinity of Ibuprofen coprecipitated with Eudragit RS100 and stated that the addition of the amorphous polymer to the drug decreased the degree of crystallinity of the latter. DSC studies showed a reduction in the peak intensity together with a slight shift at the peak location towards lower temperature of the zwitterionic polymer coprecipitated. The author concluded a significant drug-polymer interaction since the peak onset decreased from 65 to $58^{\circ} \mathrm{C}$. Dubernet et al. ${ }^{40}$ studied the DSC of ethyl cellulose microspheres containing Ibuprofen and reported the small molecules was dissolved in a polymer and plasticized the macromolecular chains which led to lowering of the glass transition temperature of the polymer. Accordingly, it can be concluded that the encapsulated drug itself acts as a genuine plasticizer for Eudragit RS100. Therefore, a part Ibuprofen molecules form a solid solution with the polymer. Then DSC study reveals that the drug exists in the Eudragit RS100 microspheres as a solid solution, as crystalline domains and as a product of a chemical interaction.

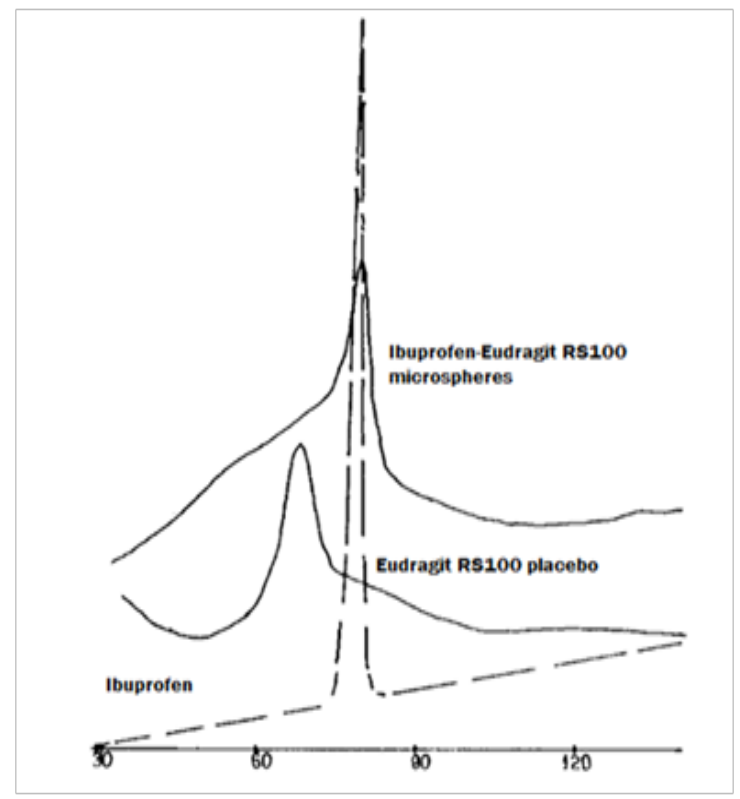

Figure 6 DSC scan of ibuprofen, eudragit RSI00 placebo microspheres and ibuprofen-eudragit RSI00 microspheres containing Ibuprofen.

Bodmeier et al. ${ }^{41}$ prepared poly (dl-lactide) micro spheres containing quinidine sulphate using a solvent evaporation technique. The drug was dissolved or dispersed in the dichloromethane polymer solution. He found that, the burst effect of the microspheres prepared by using the clear organic phase solution was more than that of the spheres prepared using dispersed drug. The author owed the initial faster drug release from micro spheres prepared by using the drug solution in the organic phase to its higher content of non-crystalline drug. Accordingly it can be concluded that the rapid initial drug release may be due to the drug crystal attached to the microspheres surfaces. In addition the solid solution form of the drug in the microspheres structure near the microsphere surface has also a role for this effect. This suggested explanation which based on surface microsphere scan and experimentally results suggested by Bodmeier et al. ${ }^{41}$ should be also supported with finding that the total amount of drug release in gastric simulating medium is nearly twice to its solubility in the same media. Currently, the observed incomplete drug release from the prepared microspheres could be interpreted in the lights of the drugpolymer physico-chemical interaction and that the interacted drug was strongly held by the polymer so that it was unable to be released.

\section{Conclusion}

From above it can be concluded that, the actual drug content of the acidic drug can increase by using both acidic aqueous phase medium and the right concentration of gelatin as an anti aggregating agent. Also it can prepare high yield of the product with narrow particle size. This restricted condition led to entrap the acidic drug in more than 
one form, solid solution, drug crystal and any kind of drug polymer interaction which will be reflected on the drug release process.

\section{Acknowledgements}

The authors acknowledge the staff of the department of Pharmaceutical technology, Faculty of Pharmacy, Tanta University for their support and encouragement in carrying out the work.

\section{Conflict of interest}

None.

\section{References}

1. Mady O, Korsatko W, Zain Eldin E. Eudragit $\mathrm{RS}_{100}$ placebo microspheres in different media, studying the morphology, surface and internal microspheres structure. International Journal of Advanced Pharmaceutics. 2015;5(1):43-55.

2. Friedman M, Seckman C, Stubbs C. Ibuprofen: pharmacology, therapeutics and side effects. Clin Pharmacol. 1990;30:64.

3. USP XVII. 1990. $682 \mathrm{p}$.

4. Wilson CG, Washington N, Greaves JL, et al. Bimodal release of drug in a sustained release Ibuprofen formulation: A scintigraphic and pharmacokinetic open study in healthy volunteers under different conditions of food intake. Int J Pharmaceut. 1989;50(2):155-161.

5. Barkai A, Pathak YV, Benita S. Polyacrylate (Eudrugit retard) microspheres for oral controlled release of nifedipine. I. Formulation design and process optimization. Drug Dev Ind Pharm. 1990;16(13):2057-2075.

6. Kawashima Y, Niwa T, Hand T, et al. Control of Prolonged Drug Release and Compression Properties of Ibuprofen Microspheres With Acrylic Polymer, Eudragit RS, by Changing Their Intraparticle Porosity. Chem Pharm Bull. 1992;40(1):196-201.

7. Devrim B, Canefe K. Preparation and evaluation of modified release ibuprofen microspheres with acrylic polymers (Eudragit) by quasiemulsion solvent diffusion method: effect of variables. Acta Pol Pharm. 2006;63(6):521-534.

8. Bolourtchian N, Karimi K, Aboofazeli R. Preparation and characterization of ibuprofen microspheres. J Microencapsul. 2005;22(5):529-538.

9. Nandini Saha, Ikramul Hasan, Mehrina Nazmi, et al. Design and Development of Sustained Release Microspheres of Ibuprofen by Emulsification Solvent Evaporation Method Using Polymeric Blend. Bangladesh Pharmaceutical Journal. 2013;16(1):39-44.

10. Sengel-Turk CT, Hascicek C, Gonul N. Ethylcellulose-based matrixtype microspheres: influence of plasticizer RATIO as pore-forming agent. AAPS PharmSciTech. 2011;12(4):1127-1135.

11. Martin A, Swerbrick J, Cammarata A. Physical Pharmacy. 3rd ed. USA; lea and Febigon, Pennsylvania; 1989. 503 p.

12. Sprockel OL, Prapaitrakul W. A comparision of microencapsulation by various emulsion techniques. Int J Pharm. 1990;58(2):123-127.

13. Bodmeier R, McGinity JW. Solvent selection in the preparation of poly (DL-lactide) microspheres prepared by the solvent evaporation method. Int J Pharm. 1988;43(1-2):179-186.

14. Gebre-Mariam T, Armstrong NA, Brain KR, et al. The effect of gelatin grade and concentration on the migration of solutes into and through glycerogelatin gels. J Pharm Pharmacol. 1989;41(8):524-527.

15. Takamura K, Koiki M, kondo T. Studies on microcapsules IX: Permeability of polyphthalamide microcapsule membranes to electrolytes. Kolloid-Z polm. 1971;248(1-2):929-933.
16. Takamura K, Koiki M, kondo T. Microcapsules XIV: Effects of membrane materials and viscosity of aqueous phase on permeability of polyamide microcapsules toward electrolytes. J Pharma Sci. 1973;62(4):610 612 .

17. Kislalioglu MS, Khan MA, Blount C, et al. Physical characterization and dissolution properties of ibuprofen:Eudragit coprecipitates. J Pharm Sci. 1991;80(8):799-804.

18. Wada R, Hyon SH, Ikada Y. Lactic acid oligomer micro-spheres containing hydrophilic drugs. J Pharm Sci. 1990;79(10):919-924.

19. Suzuki K, Price JC. Microencapsulation and dissolution properties of a neuroleptic in a biodegradable polymer, poly (d,1-lactide). J Pharm Sci. 1985;74(1):21-24.

20. Nishioka Y, Kyotani S, Okamura M, et al. Evaluation and preparation of albumen microspheres and microcapsules containing cisplatin. Chem Pharm Bull. 1989;37(5):1399-1400.

21. Nishioka Y, Kyotani S, Okamura M, et al. Release characteristic of cisplatin chitosan microspheres and effect of containing chitin. Chem Pharm Bull. 1990;38(10):2871-2873.

22. Huang HP, Ghebre-Sellassie I. Preparation of microspheres of watersoluble pharmaceuticals. J Microencapsul. 1989;6(2):219-225.

23. Ogawa Y, Yamamoto M, Okada H, et al. A new technique to efficiently entrap leuprolide acetate into microcapsules of polylactic acid or copoly (lactic/glycolic) acid. Chem Pharm Bull. 1988;36(3):1095-1003.

24. Beck LR, Cowsar DR, Lewis DH, et al. A new long-acting injectable microcapsule system for the administration of progesterone. Fertil Steril. 1979;31(5):545-551.

25. Kojima T, Nakano M, Juni K, et al. Preparation and evaluation in vitro of polycarbonate microspheres containing local anesthetics. Chem Pharm Bull. 1984;32(7):2795-2802.

26. Uchida T, Tasutake T, Goto S. Utility of mixture of commercially available polymers as constituents of sustained-release microcapsules containing cefadroxil or theophylline. Chem Pharm Bull. 1992;40(2):463466.

27. Bodmeier R, McGinity JW. Polylactic acid microspheres containing quinidine base and quinidine sulphate prepared by the solvent evaporation method. III. Morphology of the microspheres during dissolution studies. J Microencapsul. 1988;5(4):325-330.

28. Seki T, Kawaguchi T, Endoh H, et al. Controlled release of 3', 5'-diester prodrugs of 5-fluoro-2'-deoxyuridine from poly-L-lactic acid microspheres. J Pharma Sci. 1990;79(11):985-987.

29. Singh J, Robinson DH. Controlled release captopril microcapsules:effect of non-ionic surfactants on release from ethyl cellulose microcapsules. J Microencapsul. 1988;5(2):129-137.

30. Jeyanthi R, Rao P. Release characteristics of bleomycin mitomycin $C$ and 5-fluorouracil from gelatin microspheres. International $J$ of Pharmaceutics. 1989;55(1):31-37.

31. Spenlehauer G, Veilard M, Benoît JP. Formulation and characterization of cisplatin loaded poly(d,1-lactide) microspheres for chemoembolism. J Pharm Sci. 1986;75(8):750-755.

32. Babay D, Hoffman A, Benita S. Design and release kinetic pattern evaluation of indomethacin microspheres intended for oral administration. Biomaterials. 1988;9(6):482-488.

33. Juni K, Nakano M, Kubota M. Control release of aclarubicin, an anticancer antibiotic, from poly- $\beta$-hydroxybutyric microspheres. Journal of Controlled Release. 1986;4:25.

34. Armand JY, Magnard F, Bozon J, et al. Modelling of drug release in gastric liquid from spheric galenic forms with Eudragit matrix, Int $J$ of Pharmaceutics. 1987;40(1-2):33-41. 
35. Suryakusuma H, Jun HW. Encapsulated hydrophilic polymer beads containing indomethacin as controlled release drug delivery systems. $J$ Pharm Pharmacol. 1984;36(8):497-501.

36. Oth M, Moes A. $5^{\text {th }}$ international congress of pharmaceutical technology. 1989;2:104.

37. Juni K, Nakano M, Kubota M. Microencapsulation and Controlled Release of Insulin from Polylactic Acid Microcapsules. $J$ of controlled release. 1986;4:25.

38. Nakanishi K. Infrared absorption spectroscopy, practical. USA, California: Holden-day Inc; 1962. 233 p.
39. El-Hinnawi MA, Najib NM. Ibuprofen-polyvinylpyrrolidone dispersions. Proton nuclear magnetic resonance and infrared studies. International J of Pharmaceutics. 1987;37(1-2):175-177.

40. Dubernet C, Rouland JC. Benoit JP Ibuprofen-loaded ethylcellulose microspheres:analysis of the matrix structure by thermal analysis. J Pharma Sci. 1991;80(11):1029-1033.

41. Bodimer R, Thiele W, McGinity. $5^{\text {th }}$ congress international congress of pharmaceutical technology. 1989;2:69-75. 